\title{
Data Fusion Based Thermal Sensors for Mass Flow Measurement in Pneumatic Conveying
}

\author{
Hong Zhang, Wenqian Zhang, and Dan Ling \\ School of Automation, Huazhong University of Science and Technology, Wuhan 430074, China \\ Correspondence should be addressed to Hong Zhang; sunracer@mail.hust.edu.cn
}

Received 24 July 2014; Accepted 1 September 2014; Published 29 September 2014

Academic Editor: Wei Zhang

Copyright ( 2014 Hong Zhang et al. This is an open access article distributed under the Creative Commons Attribution License, which permits unrestricted use, distribution, and reproduction in any medium, provided the original work is properly cited.

\begin{abstract}
This paper mainly investigates the mass flow measurement of the gas-solid two-phase flow in pneumatic conveyor. A new data fusion method based on the thermal sensors is proposed, which can improve the overall accuracy of the flow rate of the gas-solid two-phase flow and the time resolution, that is, the overall response rate of the system. Based on this method, a model fusion used in time domain is obtained. Several examples are given to illustrate the advantages of the proposed method.
\end{abstract}

\section{Introduction}

The measurement of the gas-solid two-phase flow in pneumatic conveyor is of great significance, especially in the coilfired station and steel production industry. The solid phase concentration measurement of gas-solid two-phase flow is one of the most difficult parameters to be measured, which has always been the research focus and difficulty in industrial detection over the last decade [1]. Recently, many sensors, such as capacitance sensor used in process tomography $[2,3]$, electrostatic sensor $[4,5]$, optical sensor $[6,7]$, microwave sensor $[8,9]$, and thermal sensor $[10,11]$, have been adopted in the research on gas-solid two-phase flow. A variety of data processing methods have been used in the measurement system to improve the accuracy, including neural network technique [12,13], soft-sensing technique [14], and data fusion method [15].

The methods in thermology fell into two categories: heat balance method and heat transfer method. At present, the heat balance method, which has been employed for many years, is known as a comparatively mature measurement method. A lot of work has been done on the establishment of measurement models [16, 17], analysis of affecting error, and system implementation. Besides, several field devices with good practicality have been established. Despite its late start on the measurement of gas-solid two-phase flow, the heat transfer method is becoming popular gradually.
Based on the heat transfer method, a noninvasive prototype system measuring the mass flow rate of two-phase flow can be first found in Moriyama [11]. The system can measure the mass flow rate of gas-solid from dilute phase to dense phase. Another noninvasive method measuring the mass of gas-solid, which measures the solid phase mass flow by heating the solid particles in the tube wall and measuring the temperature distribution of gas-solid two-phase flow, is well discussed in [18]. This method is similar to the principle for measurement of thermal distribution. A heat transfer probe method which is employed to measure pulverized coal concentration of the power feeding system in a power station is proposed in [19]. This method adopts elliptic probes with different placement to measure the concentration of pulverized coal and air volume. Yuan et al. [20] place a probe with electric heating in the flow to measure the solid flow rate. There are different heat transfer effects when the probe contacts the flow media with different flow velocities, concentrations, and particle diameters. In this case, the solid phase flow in the two-phase flow, given air volume, can be measured by the electrical heating power and the measured temperature of the probe. The heat transfer method has also been applied in [21]. They adopt the principle of heat dissipation type mass flowmeter in the plug-in probe consisting of platinum thermal resistance. The control circuit works in an environment of constant difference in temperature where it has a better response characteristic. Meanwhile, the thermal 
sensor and temperature sensor in the same base can be formed into a compound transducer, which can not only acquire the flow signal but also decouple the temperature signal.

In terms of data processing technique, several key issues in indirect measurement method have been studied and discussed deeply, including spatial filtering effect, average effect, and the resolution and sensitivity of the tomographic imaging sensor array [22]. The BP artificial neural network has been used to predict the measurement of the solid flow rate of two-phase flow with heat transfer method and the prediction process is examined in [13]. Based on a lot of experimental data, it has been shown that the complicated nonlinear problem between the solid flow rate and the factors can be solved by the artificial neural network. The data fusion is attempted to deal with the measurement of heat balance method and heat transfer method via mathematical analysis, which has made some achievements [21]. Based on the result, this paper conducts further research.

The two different methods which measure gas-solid twophase flow discussed in this paper are necessary to achieve data fusion and have something in common. First of all, not only are the two methods based on the principle of heat transfer, but also they have a direct relationship with the flow rate of air. Secondly, although each method has its own advantages and disadvantages, both of the measurement data are processed by reasonable data fusion method, which can improve the measurement accuracy, response speed, and robustness of the system.

The rest of the paper is organized as follows. Section 2 gives some problems in data fusion method and real-time measurement. Section 3 introduces the general model fusion method. Section 4 provides some main results. And Section 5 discusses the window size and the distribution of weights. The final section gives the conclusion of this paper.

\section{Date Fusion Based Real-Time Measurement}

For high performance, the measurement system has to provide not only high accuracy measured output information, but also a sufficiently high response speed in industrial process control, such as the boiler combustion control. This paper mainly investigates the real-time measurement via data fusion method, which can improve the overall accuracy of the flow rate of the gas-solid two-phase flow and the time resolution, that is, the overall response rate of the system. Date fusion based real-time measurement can be described as shown in Figure 1.

In Figure 1, when the sample data of system B with higher time resolution is measured in real-time, the corresponding sample data of system A could not be generated to fuse the data and modify the data of system $\mathrm{B}$ in time. If the window size is $N$, there will be a sample data $\widetilde{X}_{1}$ in $[N / 2]$. In general, the data will be delayed in half of the window size and when the processing window is moving forward, $\widetilde{X}_{1}$ cannot stay in the correct place. The sample interval of $\widetilde{X}_{1}$ is too long to ensure that the sample value of $\widetilde{X}_{1}$ is proper in the processing window.
The sample time of heat balance method is about two minutes while the sample time of heat transfer method is five seconds because the probe of heat transfer method is placed in the fluid directly and the sensor has a speedy response. If the window size is set to be 16 , the sampling point will not be inside some windows when the window is moving. If the window size is very large, the correlation among the measurements from the two measured methods will be reduced very much. If the sampling point is not in the middle of the window, it is difficult to satisfy the assumption of weighted average.

It is suggested that the measurements are processed by data fusion method after the sample value $\widetilde{X}$ with high accuracy is predicted by the preliminary data if the measurements are processed in real-time. For one sample value, we conclude

$$
\widetilde{X}_{1} \approx \sum_{i=1}^{n} w_{1, i} \cdot x_{i}
$$

where $\widetilde{X}$ expresses the weighted average of $\tilde{x}$. $\widetilde{X}$ can be predicted by the modified sample value of system $B$.

After the sample value of system B is corrected, a new sample value $X_{n+1}$ will be put into the processing window and the old sample value $X_{1}$ will be removed from the window. $X_{1}$ is the corrected sample value while $X_{n+1}$ has not been corrected. In this paper, the estimate of $\widetilde{X}$ can be obtained by predicting the corrected measurement of $X_{n+1}$ as follows:

$$
\widetilde{X}_{1}^{\prime}=\widetilde{X}_{1}-w_{1,1} x_{1}+w_{1, n} \widehat{x}_{n+1}
$$

where $\widehat{x}_{n+1}$ is the $(n+1)$ th estimate which is predicted by the corrected measurement of system B.

\section{Data Fusion}

In the data fusion method [23], there are several methods to get the measured value of the same measurement $x$. Denote the $i$ th measurement value of $x$ as $\tilde{x}^{(i)}, i=1, \ldots, n$. Then, the corresponding measured error can be written as

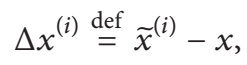

where $x$ represents the actual value of the measurement and $\Delta x^{(i)}$ is the measured error of the $i$ th measured method.

If each measured error obeys normal distribution with zero mean and standard deviation $\sigma^{(i)}$, the probability density function of $\Delta x^{(i)}$ can be described as

$$
\begin{aligned}
f(x)= & \frac{1}{\sqrt{2 \cdot \pi} \cdot \sigma^{(i)}} \cdot \exp \left(-\frac{\left(\Delta x^{(i)}\right)^{2}}{2 \cdot\left(\sigma^{(i)}\right)^{2}}\right)=\frac{1}{\sqrt{2 \cdot \pi} \cdot \sigma^{(i)}} \\
& \cdot \exp \left(-\frac{\left(\tilde{x}^{(i)}-x\right)^{2}}{2 \cdot\left(\sigma^{(i)}\right)^{2}}\right) .
\end{aligned}
$$


If the measured error $\Delta x^{(i)}$ is independent, $\rho(x)$ expresses the joint probability destiny of $x$ and it can be written as

$$
\begin{aligned}
\rho(x) & =\prod_{i=1}^{n} \frac{1}{\sqrt{2 \cdot \pi} \cdot \sigma^{(i)}} \cdot \exp \left(-\frac{\left(\tilde{x}^{(i)}-x\right)^{2}}{2 \cdot\left(\sigma^{(i)}\right)^{2}}\right) \\
& =\left(\prod_{i=1}^{n} \frac{1}{\sqrt{2 \cdot \pi} \cdot \sigma^{(i)}}\right) \cdot \exp \left(-\sum_{i=1}^{n} \frac{\left(\widetilde{x}^{(i)}-x\right)^{2}}{2 \cdot\left(\sigma^{(i)}\right)^{2}}\right) .
\end{aligned}
$$

It is reasonable to select the maximum likelihood estimate as the estimate of $x$ when the probability $\rho(x)$ is the largest. Since $\exp (z)$ is an increasing function, maximizing $\rho(x)=$ $A \cdot \exp (-B(x))$ is equivalent to minimizing $B(x)$, and then we can derive that

$$
\min \left(\sum_{i=1}^{n} \frac{\left(\tilde{x}^{(i)}-x\right)^{2}}{2 \cdot\left(\sigma^{(i)}\right)^{2}}\right) .
$$

Differentiating (6) with respect to $x$ and equating the derivative to zero, we can conclude the maximum value:

$$
x=\frac{\sum_{i=1}^{n} \tilde{x}^{(i)} \cdot\left(\sigma^{(i)}\right)^{-2}}{\sum_{i=1}^{n}\left(\sigma^{(i)}\right)^{-2}} .
$$

The accuracy of this fused estimator can be described by the synthetic standard deviation $\sigma$ as follows:

$$
\sigma^{-2}=\sum_{i=1}^{n}\left(\sigma^{(i)}\right)^{2}
$$

It is assumed that there are two measurement systems, that is, the heat balance method and the heat transfer method, of which the accuracy and time (spatial) resolution are different. The accuracy of system with higher time resolution is higher, while the accuracy of system with lower time resolution is lower. Considering the individual and the mutual probability distribution, the data fusion method will use least squares (LS) algorithm to minimizing the deviation of the fused data.

In convenience, $\widetilde{X}_{j}$ is defined as the sample value (or estimated value) of system with low time resolution and high accuracy and the corresponding system is called system A. $\tilde{x}_{i}$ expresses the sample value (or estimated value) of system with high time resolution and high accuracy and the corresponding system is system B. In this paper, system A uses the heat balance method and system B adopts the heat transfer method. rithm.

The following problems will be considered in this algo-

(A) Each sampling value $\tilde{x}_{i}$ from system B is approximately equal to the actual value $x_{i}$ at a certain probability in the corresponding time, with the standard deviation $\sigma_{h, i}$ :

$$
\tilde{x}_{i} \approx x_{i} i
$$

(B) Each sampling value $\widetilde{X}_{j}$ from system $\mathrm{A}$ with a high time resolution is approximately equal to the weighted average of the actual values, with the standard deviation $\sigma_{l, i}$ :

$$
\widetilde{X}_{j} \approx \sum_{i} w_{j, i} \cdot x_{i},
$$

where $w_{j, i} \geq 0$ and $\sum_{i=1}^{n} w_{j, i}=1$. In general, the weights are known. For example, these weights are equal in a joint inversion system and we get

$$
\widetilde{X}_{j} \approx \frac{x_{i}(1, j)+\cdots+x_{i}\left(k_{j}, j\right)}{k_{j}} .
$$

In this application, the influences on weights are different because the sampling intervals are different. So the weights will be different and the problem about the selection of weight will be discussed in the following sections.

(C) A prior estimate: if the system B has a prior estimate with higher time resolution, we get

$$
x_{i} \approx x_{\mathrm{pr}, i}
$$

where the standard deviation of $x_{\mathrm{pr}, i}$ is $\sigma_{\mathrm{pr}, i}$. In application, if there is no prior estimate, we can set $\sigma_{\mathrm{pr}, i}=\infty$ and its influence can be eliminated.

(D) The sampling value $\widetilde{X}_{j}$ from system $\mathrm{A}$ is approximately equal to the actual value at the sampling time point with a high resolution at a certain probability, with the accuracy corresponding to the standard deviation $\sigma_{e, j}$ :

$$
\widetilde{X}_{j} \approx x_{i}(l, j) .
$$

The expectation can be estimated by the sampling value of system B:

$$
E_{j}=\frac{1}{k_{j}} \cdot \sum_{l=1}^{k_{j}} \widetilde{x}_{i}(l, j) .
$$

The corresponding standard deviation is defined as $\sigma_{e, j}$ and it can be expressed as

$$
\sigma_{e, j}^{2}=\frac{1}{k_{j}} \cdot \sum_{l=1}^{k_{j}}\left(\widetilde{x}_{i}(l, j)-E_{j}\right)^{2} .
$$

Next, the least squares (LS) technique will be applied to combine these approximate equalities and find the desirable estimate of the measurements by minimizing the resulting sum of weighted squared differences.

According to the LS approach, the sum of the different deviation in the above expression is minimized. In general case, we minimize the following expression:

$$
\begin{aligned}
& \sum_{i=1}^{n} \frac{\left(x_{i}-\widetilde{x}_{i}\right)^{2}}{\sigma_{h, i}^{2}}+\sum_{j=1}^{m} \frac{1}{\sigma_{l, j}^{2}}\left(\widetilde{X}_{j}-\sum_{i=1}^{n} w_{j, i} \cdot x_{i}\right)^{2} \\
& +\sum_{i=1}^{n} \frac{\left(x_{i}-x_{\mathrm{pr}, i}\right)^{2}}{\sigma_{\mathrm{pr}, i}^{2}}+\sum_{j=1}^{m} \sum_{l=1}^{k_{j}} \frac{\left(\widetilde{X}_{j}-x_{i}(l, j)\right)^{2}}{\sigma_{e, j}^{2}} .
\end{aligned}
$$


The solution of the LS approach includes the deviation of the measurements with high resolution (the first expression), the deviation of the prior estimate with high resolution (the third expression), the deviation of the weighted estimates of which low resolution are weighted by the sampling value of the high resolution (the fourth expression), and the deviation between the sampling value of high resolution and the corresponding area with the high resolution (the second expression). The synthesis results minimize the overall deviation in the above and the solution of the estimated $x_{i}$ is equal to the value of data fusion.

Differentiation with respect to $x_{i}$ leads to the following system of linear equations:

$$
\begin{gathered}
\frac{1}{\sigma_{h, i}^{2}} \cdot\left(x_{i}-\widetilde{x}_{i}\right)+\sum_{j: j \ni i} \frac{1}{\sigma_{l, j}^{2}} \cdot w_{j, i} \cdot\left(\sum_{i^{\prime}=1}^{n} w_{j, i^{\prime}} \cdot x_{i^{\prime}}-\widetilde{X}_{j}\right) \\
+\frac{1}{\sigma_{\mathrm{pr}, i}^{2}} \cdot\left(x_{i}-x_{\mathrm{pr}, i}\right)+\sum_{j: j \ni i} \frac{1}{\sigma_{e, j}^{2}} \cdot\left(x_{i}-\widetilde{X}_{j}\right)=0,
\end{gathered}
$$

where $j \ni i$ means that the $j$ th sampling value corresponding to a model with a low spatial resolution covers the $i$ th sampling value.

In the above solution of LS approach, the prior estimate $x_{\mathrm{pr}, i}$ and the estimate $\tilde{x}_{i}$ from system A with a higher resolution have the general form. Then, they can be expressed into a unified form:

$$
\frac{1}{\sigma_{h, i}^{2}} \cdot\left(x_{i}-\tilde{x}_{i}\right)+\frac{1}{\sigma_{\mathrm{pr}, i}^{2}} \cdot\left(x_{i}-x_{\mathrm{pr}, i}\right)
$$

Equation (18) can be expressed as follows:

$$
\sigma_{f, i}^{-2} \cdot\left(x_{i}-x_{f, i}\right)
$$

where

$$
\begin{gathered}
x_{f, i} \stackrel{\text { def }}{=} \frac{\tilde{x}_{i} \cdot \sigma_{h, i}^{-2}+x_{\mathrm{pr}, i} \cdot \sigma_{\mathrm{pr}, i}^{-2}}{\sigma_{h, i}^{-2}+\sigma_{\mathrm{pr}, i}^{-2}} \\
\sigma_{f, i}^{-2} \stackrel{\text { def }}{=} \sigma_{h, i}^{-2}+\sigma_{\mathrm{pr}, i}^{-2} .
\end{gathered}
$$

Similarly, if the standard deviation is set to infinity, the influence of the corresponding term will be eliminated. For example, we can set $\sigma_{h, i}=0$ if only the prior estimates of the system are known.

After simplification, the LS expression is

$$
\begin{aligned}
& \sigma_{f, i}^{-2} \cdot\left(x_{i}-x_{f, i}\right)+\sum_{j: j \ni i} \frac{1}{\sigma_{l, j}^{2}} \cdot w_{j, i} \cdot\left(\sum_{i^{\prime}=1}^{n} w_{j, i^{\prime}} \cdot x_{i^{\prime}}-\widetilde{X}_{j}\right) \\
& +\sum_{j: j \ni i} \frac{1}{\sigma_{e, j}^{2}} \cdot\left(x_{i}-\widetilde{X}_{j}\right)=0 .
\end{aligned}
$$

Now, we consider the case of a single estimate with low resolution.
Let us consider the simplest case, when we have exactly one estimate from system A with a low resolution. In this case, (21) takes the following form:

$$
\begin{aligned}
& \sigma_{f, i}^{-2} \cdot\left(x_{i}-x_{f, i}\right)+\frac{1}{\sigma_{l, 1}^{2}} \cdot w_{1, i} \cdot\left(\sum_{i^{\prime}} w_{1, i^{\prime}} \cdot x_{i^{\prime}}-\widetilde{X}_{1}\right) \\
& +\frac{1}{\sigma_{e, 1}^{2}}\left(x_{i}-\widetilde{X}_{1}\right)=0 .
\end{aligned}
$$

If multiplying both sides of (22) by $\sigma_{f, i}^{2}$, we conclude that

$$
\begin{gathered}
x_{i}-x_{f, i}+\frac{\sigma_{f, i}^{2}}{\sigma_{l, 1}^{2}} \cdot w_{1, i} \cdot\left(\sum_{i^{\prime}} w_{1, i^{\prime}} \cdot x_{i^{\prime}}-\widetilde{X}_{1}\right) \\
+\frac{\sigma_{f, i}^{2}}{\sigma_{e, 1}^{2}} \cdot\left(x_{i}-\widetilde{X}_{1}\right)=0 .
\end{gathered}
$$

If we introduce an auxiliary variable $\mu$ as

$$
\mu \stackrel{\text { def }}{=} \frac{1}{\sigma_{l, 1}^{2}} \cdot\left(\sum_{i^{\prime}} w_{1, i^{\prime}} \cdot x_{i^{\prime}}-\widetilde{X}_{1}\right) \text {, }
$$

we get the equation

$$
x_{i}-x_{f, i}+w_{1, i} \cdot \sigma_{f, i}^{2} \cdot \mu+\frac{\sigma_{f, i}^{2}}{\sigma_{e, 1}^{2}} \cdot\left(x_{i}-\widetilde{X}_{1}\right)=0 .
$$

By keeping terms proportional to $x_{i}$ in the left-hand side and by moving all the other terms to the right-hand side, we get

$$
\left(1+\frac{\sigma_{f, i}^{2}}{\sigma_{e, 1}^{2}}\right) \cdot x_{i}=x_{f, i}-w_{1, i} \cdot \sigma_{f, i}^{2} \cdot \mu+\frac{\sigma_{f, i}^{2}}{\sigma_{e, 1}^{2}} \cdot \widetilde{X}_{1}
$$

Hence

$$
\begin{aligned}
x_{i}= & \frac{x_{f, i}}{1+\sigma_{f, i}^{2} / \sigma_{e, 1}^{2}}-\frac{w_{1, i} \cdot \sigma_{f, i}^{2}}{1+\sigma_{f, i}^{2} / \sigma_{e, 1}^{2}} \cdot \mu+\widetilde{X}_{1} \\
& \cdot \frac{\sigma_{f, i}^{2} / \sigma_{e, 1}^{2}}{1+\sigma_{f, i}^{2} / \sigma_{e, 1}^{2}} .
\end{aligned}
$$

According to the definition of $\mu$, it includes the actual value of random variable $x_{i}$. To make this expression practical, we must describe $\mu$ in terms of the given $\widetilde{x}_{i}$ and $\widetilde{X}_{1}$.

From (27), we get

$$
\begin{aligned}
\sum_{i=1}^{n} w_{1, i} \cdot x_{i}= & \sum_{i=1}^{n} \frac{w_{1, i} \cdot x_{f, i}}{1+\sigma_{f, i}^{2} / \sigma_{e, 1}^{2}}-\mu \\
& \cdot \sum_{i=1}^{n} \frac{w_{1, i}^{2} \cdot \sigma_{f, i}^{2}}{1+\sigma_{f, i}^{2} / \sigma_{e, 1}^{2}}+\widetilde{X}_{1} \cdot \sum_{i=1}^{n} \frac{w_{1, i} \cdot\left(\sigma_{f, i}^{2} / \sigma_{e, 1}^{2}\right)}{1+\sigma_{f, i}^{2} / \sigma_{e, 1}^{2}} .
\end{aligned}
$$


According to definition of $\mu$, we conclude that

$$
\begin{aligned}
\sigma_{l, 1}^{2} \cdot \mu= & \sum_{i=1}^{n} w_{1, i} \cdot x_{i}-\widetilde{X}_{1} \\
= & \sum_{i=1}^{n} \frac{w_{1, i} \cdot x_{f, i}}{1+\sigma_{f, i}^{2} / \sigma_{e, 1}^{2}}-\mu \cdot \sum_{i=1}^{n} \frac{w_{1, i}^{2} \cdot \sigma_{f, i}^{2}}{1+\sigma_{f, i}^{2} / \sigma_{e, 1}^{2}}+\widetilde{X}_{1} \\
& \cdot \sum_{i=1}^{n} \frac{w_{1, i} \cdot\left(\sigma_{f, i}^{2} / \sigma_{e, 1}^{2}\right)}{1+\sigma_{f, i}^{2} / \sigma_{e, 1}^{2}}-\widetilde{X}_{1} .
\end{aligned}
$$

According the definition of weighted average, the sum of the weights is equal to one:

$$
\sum_{i=1}^{n} w_{1, i}=1
$$

Then the final two terms in the left-hand side of (29) can be merged into

$$
\begin{aligned}
& \widetilde{X} \cdot \sum_{i=1}^{n} \frac{w_{1, i} \cdot\left(\sigma_{f, i}^{2} / \sigma_{e, 1}^{2}\right)}{1+\sigma_{f, i}^{2} / \sigma_{e, 1}^{2}}-\widetilde{X}_{1} \\
& =\widetilde{X}_{1} \cdot\left(\sum_{i=1}^{n} w_{1, i} \frac{\sigma_{f, i}^{2} / \sigma_{e, 1}^{2}}{1+\sigma_{f, i}^{2} / \sigma_{e, 1}^{2}}-\sum_{i=1}^{n} w_{1, i}\right) \\
& =-\widetilde{X}_{1} \cdot \sum_{i=1}^{n} \frac{w_{1, i}}{1+\sigma_{f, i}^{2} / \sigma_{e, 1}^{2}} .
\end{aligned}
$$

By taking (31) into (24) and moving all terms containing $\mu$ to the left-hand side, we get

$$
\begin{aligned}
& \mu \cdot\left(\sigma_{l, 1}^{2}+\sum_{i=1}^{n} \frac{w_{1, i}^{2} \cdot \sigma_{f, i}^{2}}{1+\sigma_{f, i}^{2} / \sigma_{e, 1}^{2}}\right) \\
& =\sum_{i=1}^{n} \frac{w_{1, i} \cdot x_{f, i}}{1+\sigma_{f, i}^{2} / \sigma_{e, 1}^{2}}-\widetilde{X}_{1} \cdot \sum_{i=1}^{n} \frac{w_{1, i}}{1+\sigma_{f, i}^{2} / \sigma_{e, 1}^{2}} \\
& =\sum_{i=1}^{n} \frac{w_{1, i}\left(x_{f, i}-\widetilde{X}_{1}\right)}{1+\sigma_{f, i}^{2} / \sigma_{e, 1}^{2}} .
\end{aligned}
$$

If we introduce two auxiliary variables $N$ and $D$, the auxiliary variable $\mu$ can be computed as follows:

$$
\mu=\frac{N}{D}
$$

where

$$
\begin{gathered}
D=\left(\sigma_{l, 1}^{2}+\sum_{i=1}^{n} \frac{w_{1, i}^{2} \cdot \sigma_{f, i}^{2}}{1+\sigma_{f, i}^{2} / \sigma_{e, 1}^{2}}\right) \\
N=\sum_{i=1}^{n} \frac{w_{1, i}\left(x_{f, i}-\widetilde{X}_{1}\right)}{1+\sigma_{f, i}^{2} / \sigma_{e, 1}^{2}} .
\end{gathered}
$$

Then, under higher time resolution, we compute the more accurate estimate for $x_{i}, i=1, \ldots, h$, as

$$
\begin{aligned}
\widehat{x}_{i}= & \frac{x_{f, i}}{1+\sigma_{f, i}^{2} / \sigma_{e, 1}^{2}}-\frac{w_{1, i} \cdot \sigma_{f, i}^{2}}{1+\sigma_{f, i}^{2} / \sigma_{e, 1}^{2}} \cdot \mu+\widetilde{X}_{1} \\
& \cdot \frac{\sigma_{f, i}^{2} / \sigma_{e, 1}^{2}}{1+\sigma_{f, i}^{2} / \sigma_{e, 1}^{2}} .
\end{aligned}
$$

Equation (35) indicates that the sampling accuracy of the low accuracy system $B$ will be improved by modifying the sampling value with the sampling value of higher accuracy system A if the noises of system A and B obey a Gaussian distribution and the standard deviation of system $A$ is known.

The above method, using LS algorithm, shows the basic results by modifying the sampling value from a measurement system with a low accuracy when we have one estimate from system A with a higher accuracy. If all of the standard deviations in the resulting expression can be computed online or known in advance, they can be used to improve the accuracy of the system with a low accuracy.

We then solve the problem which is how to obtain the standard deviation of the above model in real-time. If this problem can be solved, the whole system will satisfy the need of the real-time measurement and this method will be applied into the polynomial prediction filter approach in the next section.

\section{Main Results}

4.1. Polynomial Prediction Filtering Method Based on FIR Scheme. In theory, the signal can be described by a $M$-order polynomial. Consider

$$
x(n)=a_{0}+a_{1} n+\cdots+a_{M} n^{M}=\sum_{l=0}^{M} a_{l} n^{l} .
$$

The polynomial filter method is that the measured signals are predicted and filtered by polynomial. A polynomial prediction filter method based on the finite impulse response (FIR) scheme has been proposed in Henry and Clarke [24]. And it is called H-N FIP predictor. This method can predict the signals with smoothness.

It is assumed that the first $n$ measurements are known and the $(n+p)$ th measurement can be predicted as follows:

$$
\widehat{x}(n+p)=\sum_{k=0}^{N-1} h(k) x(n-k),
$$

where $p$ is the prediction horizon, which can be an integer or a decimal. $h(k)$ is a coefficient of the FIR scheme. 
By taking (36) into (37), we can get

$$
\begin{gathered}
\widehat{x}(n+p)=\sum_{l=0}^{M} a_{l}[n+p]^{l}, \\
\sum_{k=0}^{N-1} h(k) x(n-k)=\sum_{k=0}^{N-1} h(k) \sum_{l=0}^{M} a_{l}[n-k]^{l}, \\
\sum_{l=0}^{M} a_{l}[n+p]^{l}=\sum_{k=0}^{N-1} h(k) \sum_{l=0}^{M} a_{l}[n-k]^{l} .
\end{gathered}
$$

Both sides of (40) are expended by the $l$ th power subentry, respectively. The polynomial which includes $a_{l}$ is that

$$
a_{l}[n+p]^{l}=\sum_{k=0}^{N-1} h(k) a_{l}[n-k]^{l}=a_{l} \sum_{k=0}^{N-1} h(k)[n-k]^{l} .
$$

If $a_{l}$ is removed from (41), we can get

$$
[n+p]^{l}=\sum_{k=0}^{N-1} h(k)[n-k]^{l}
$$

or it can expressed as

$$
[-p]^{l}=\sum_{k=0}^{N-1} h(k)[k]^{l}
$$

Equation (43) is one of the restrictions of $h(k)$.

The noise of signals can be sufficiently suppressed by minimizing the gain of the filter noise. If the noise is a white noise, the gain of signals for the digital filter based on FIR scheme can be expressed as

$$
G_{\text {noise }}=\sum_{k=0}^{N-1}|h(k)|^{2}
$$

If the restriction is satisfied, we can minimize $G_{\text {noise }}$ and the optimal solution of $h(k)$ can be obtained.

When $p=1$ and $M=1$, we can get

$$
h(k)=\frac{4 N-6 k-4}{N(N-1)} .
$$

When $p=1$ and $M=2$, we can get

$$
h(k)=\frac{9 N^{2}+(9-36 k) N+30 k^{2}-18 k+6}{N^{3}-3 N^{2}+2 N} .
$$

According to (45) and (46), the coefficient of polynomial prediction filter is independent of $x(k)$. But it is simply a matter of the window size $N$, the order $M$ of polynomial, and the prediction horizon $p$. All of the parameters are determined before computation. Therefore, this method is called one-step-prediction algorithm and it is applicable under the real-time condition.
4.2. Model Parameters Modification under the Prediction Model. In this paper, the measurements of heat balance method in data fusion are estimated. Therefore, there is a necessity of modifying the estimated signal parameter in order to adapt to the model fusion method. Once the prediction method is applied, we should consider whether all of the standard deviations defined in model fusion method need to be reestimated.

It is assumed that the corresponding standard deviations are known in a "real" fusion which is based on the actual measurements from heat balance method. The standard deviations include the standard deviation of the measurements with heat balance method and standard deviation of single measurement with heat transfer method. Both of them can be measured and calculated by experiments.

According to the principle of combination of error, assuming each measurement is independent,

$$
\sigma_{y}^{2}=\left(\frac{\partial f}{\partial x_{1}}\right)^{2} \cdot \sigma_{x_{1}}^{2}+\left(\frac{\partial f}{\partial x_{2}}\right)^{2} \cdot \sigma_{x_{2}}^{2}+\cdots+\left(\frac{\partial f}{\partial x_{n}}\right)^{2} \cdot \sigma_{x_{n}}^{2}
$$

For one estimate of system A, we have

$$
\widetilde{X}_{1}^{\prime}=\widetilde{X}_{1}-w_{1,1} x_{1}+w_{1, n} \widehat{x}_{n+1} .
$$

The standard deviation can be computed as follows:

$$
\sigma_{\widetilde{X}^{\prime}}^{2}=\sigma_{\widetilde{X}_{1}}^{2}+\sigma_{x_{1}}^{2}+\sigma_{x_{n+1}}^{2},
$$

where $\sigma_{\widetilde{X}_{1}}$ is the sampling standard deviation of one estimate for system $\mathrm{A} ; \sigma_{x_{1}}$ is the standard deviation of modified measurement for system $\mathrm{B}$ in the fusing window; and $\sigma_{x_{n+1}}$ is the standard deviation of the $(n+1)$ th modified measurement for system B. $\sigma_{x_{1}}$ can be obtained from (15).

In the polynomial prediction algorithm, the standard deviation of $\widehat{x}_{n+1}$ can be expressed as

$$
\sigma_{n+1}^{2}=\sigma_{i}^{2} \sum_{i=1}^{k} h_{0}(i)^{2}
$$

where $\sigma_{i}$ is the sample deviation.

By taking (49) into (50), the standard deviation of measurements from heat balance method can be estimated before model fusion.

\section{Discussion}

5.1. Window of the Data Fusion. The measurements of heat transfer method are estimated by polynomial prediction method; then the estimated value of heat balance method can be obtained. The estimated value will be used in the data fusion method.

Considering the polynomial prediction filtering method and the characteristic of signal, $M$ is 2 and $N$ is 16 , and $\widetilde{X}$ can be estimated from (36) in most of the windows. The measured value of heat transfer method will be modified after the actual measurement enters into the window. 
TABLE 1: The distribution of weights.

\begin{tabular}{|c|c|c|c|c|c|c|c|c|c|c|c|c|c|c|c|c|}
\hline \multirow{2}{*}{$m$} & \multicolumn{16}{|c|}{$i$} \\
\hline & 1 & 2 & 3 & 4 & 5 & 6 & 7 & 8 & 9 & 10 & 11 & 12 & 13 & 14 & 15 & 16 \\
\hline 16 & 0.5 & 0.8 & 1.1 & 1.5 & 2 & 2.5 & 3 & 4 & 4 & 3 & 2.5 & 2 & 1.5 & 1.1 & 0.8 & 0.5 \\
\hline 15 & 0.8 & 1.1 & 1.5 & 2 & 2.5 & 3 & 4 & 4 & 3 & 2.5 & 2 & 1.5 & 1.1 & 0.8 & 0.5 & 0.5 \\
\hline 14 & 1.1 & 1.5 & 2 & 2.5 & 3 & 4 & 4 & 3 & 2.5 & 2 & 1.5 & 1.1 & 0.8 & 0.8 & 0.5 & 0.5 \\
\hline 13 & 1.5 & 2 & 2.5 & 3 & 4 & 4 & 3 & 2.5 & 2 & 1.5 & 1.1 & 1.1 & 0.8 & 0.8 & 0.5 & 0.5 \\
\hline
\end{tabular}

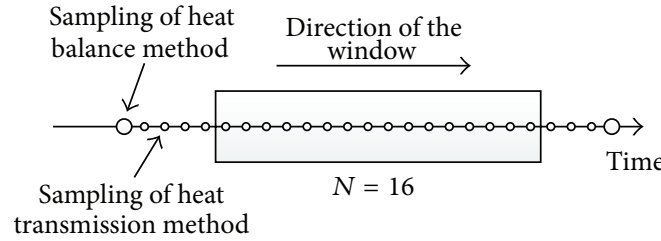

(a) The sampling of heat balance method is outside the window

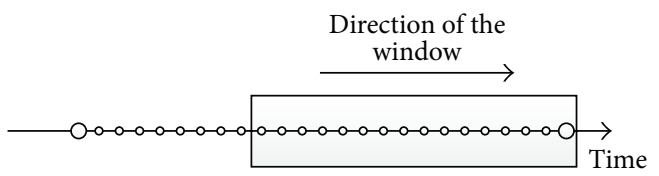

(b) The sampling of heat balance method is inside the window

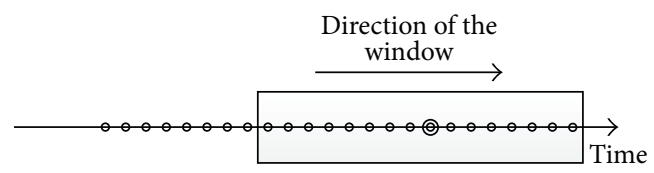

(c) The sampling of heat balance method is in the middle of the window

Figure 1: Window of data fusion.

The window adopted in this data fusion method is shown in Figure 1. Here, the sample time of the heat balance method is 2 minutes while the sample time of the heat transfer method can be 5 seconds which is much shorter than heat balance method. Therefore, there is no actual high accuracy sample value of heat balance method in the fusing window when the window is 16 . As described in the previous section, the data used in the fusing window will be estimated by the polynomial prediction filtering method.

5.2. Distribution of Weight. In Figure 1, the actual sample value rather than the estimated value will be used to process by the data fusion method once a new sample value of heat balance method enters into the window. The new sample value of heat balance method stays in the window for a while and the position of the sampling point will change.

If the sampling point of heat balance method is in middle of the fusing window, the result is the average of the sampling value of heat balance in the window which can be seen in (11). In fact, the sampling point is often not in the middle of the fusing window where the weights should be assigned reasonably.

If the window size is 16 , the weight $w_{1, i}$ of data fusion method can be expressed as follows:

$$
w_{1, i}=\frac{w_{m, i}}{16}
$$

where $i$ is the position of the sample value of system B in the fusing window. The position of the sample value of system
A in the window is used here and it satisfies the following equation:

$$
\sum_{m=1}^{16} w_{m, i}=16
$$

When a new sample value of system A enters into the window, it is reasonable that the new sample value contains sixteen sample values of system B. In other words, it is a sample value which delays half of the window. When the position of the sampling value of system $A$ is less than 13 in the window, its effect becomes weaker and the sampling value of system A will be decided by the prediction method which is used in the data fusion. The distribution of weights is shown in Table 1.

\section{Conclusion}

This paper investigates the measurement of the gas-solid twophase flow in pneumatic conveyor. A new approach called data fusion based real-time measurement is proposed, which can improve the overall accuracy of the flow rate of the gassolid two-phase flow and the time resolution. The proposed method also can be applied into the polynomial prediction filter for modifying the model parameters.

\section{Conflict of Interests}

The authors declare that there is no conflict of interests regarding the publication of this paper. 


\section{References}

[1] Y. Yan, "Mass flow measurement of bulk solids in pneumatic pipelines," Measurement Science \& Technology, vol. 7, no. 12, pp. 1687-1706, 1996.

[2] R. Deloughry and E. Pickup, "Investigation of the closed loop control of a pneumatic conveying system using tomographic imaging," in Process Imaging for Automatic Control, vol. 4188 of Proceedings of SPIE, pp. 103-113, November 2000.

[3] F. Q. Shao, Z. X. Lu, E. G. Wu, and S. Wang, "Study and industrial evaluation of mass flow measurement of pulverized coal for iron-making production," Flow Measurement and Instrumentation, vol. 11, no. 3, pp. 159-163, 2000.

[4] J. Ma and Y. Yan, "Design and evaluation of electrostatic sensors for the measurement of velocity of pneumatically conveyed solids," Flow Measurement and Instrumentation, vol. 11, no. 3, pp. 195-204, 2000.

[5] J. B. Gajewski, "Non-contact electrostatic flow probes for measuring the flow rate and charge in the two-phase gas-solids flows," Chemical Engineering Science, vol. 61, no. 7, pp. 22622270, 2006.

[6] E. Chenglin, C. Lu, C. Xu, J. Gao, and M. Shi, "A new method for measurement of local solid flux in gas-solid two-phase flow," Chinese Journal of Chemical Engineering, vol. 11, no. 6, pp. 617621, 2003.

[7] L. Aísa, J. A. Garcia, L. M. Cerecedo, I. García Palacín, and E. Calvo, "Particle concentration and local mass flux measurements in two-phase flows with PDA. Applications to a study on the dispersion of spherical particles in a turbulent air jet," International Journal of Multiphase Flow, vol. 28, no. 2, pp. 301324,2002

[8] T. G. Evans, N. G. Cutmore, E. Zoud, and F. J. Paolani, "Microwave techniques for on-line determination of pulverised coal mass flow," in Proceedings of the AMPC Asia-Pacific Microwave Conference, vol. 1, pp. 559-562, 1992.

[9] S. Bhusarapu, M. Al-Dahhan, and M. P. Dudukovic, "Quantification of solids flow in a gas-solid riser: single radioactive particle tracking," Chemical Engineering Science, vol. 59, no. 2223, pp. 5381-5386, 2004.

[10] T. Moriyama, S. Fujii, K. Abe, and M. Kobayashi, "Mass flowmeter using heat transfer for dense phase solid gas twophase flow," in Proceedings of the International Conference on Fluid Control and Measurement, vol. 33, pp. 1063-1066, 1985.

[11] T. Moriyama, "Mass flow measurement using heat transfer method for stand pipe," in Proceedings of the SICE, vol. 25, pp. 1063-1066, 1992.

[12] X. Wu and Z.-L. Yun, "Neural networks for on-line prediction of the solid flowrate in gas-solid two phase flow based on heat transfer," Boiler Technology, vol. 32, no. 12, pp. 8-11, 2001.

[13] S.-Z. Wu, Y.-J. Han, W.-X. Pu et al., "Investigation on prediction of solid flow-rate in gas-solid two phase by BP neural networks," Power System Engineering, vol. 19, no. 5, pp. 56-58, 2003.

[14] C. Xu, Y. Zhao, J. Huang et al., “The soft sensor model for mass flow rate measurement of pneumatically conveyed solids based on the artificial neural network," Acta Metrologica Sinica, vol. 27, no. 3, pp. 246-249, 2006.

[15] B. Menz, "Vortex flowmeter with enhanced accuracy and reliability by means of sensor fusion and self-validation," Measurement, vol. 22, no. 3-4, pp. 123-128, 1997.

[16] W. G. Pan and J. M. Cao, "Study on techniques for online measurement to air and pulverized coal mixture flow parameters of coal-fired boiler in power plant," Proceedings of the CSEE, vol. 24, no. 10, pp. 193-195, 2004.

[17] N. J. Zhou, Y. Q. Xue, and F. Q. Wang, "Improved energy balance method for monitoring the pulverized coal and air flow," Thermal Power Generation, vol. 34, no. 12, pp. 59-62, 2005.

[18] Y. Zheng, J. R. Pugh, D. McGlinchey, and R. O. Ansell, "Simulation and experimental study of gas-to-particle heat transfer for non-invasive mass flow measurement," Measurement, vol. 41, no. 4, pp. 446-454, 2008.

[19] L. Liu, Y. Wang, F. D. Zhou, and H. Qian, "Measurement of air flow and concentration of pulverized coal with two ellipseshaped thermal probes," Journal of Xi'an Jiaotong University, vol. 42, no. 1, pp. 23-26, 2008.

[20] Z. L. Yuan and Z. J. Lu, "Heat transfer method for solid flow rate measurement in gas-solid two phase flow," Journal of Southeast University, vol. 13, no. 2, pp. 73-77, 1997.

[21] C. X. Li, Y. Shao, and W. Li, "Measuring gas-solid two-phase mass flow based on thermal sensors fusion," Journal Huazhong University of Science and Technology, vol. 29, no. 8, pp. 50-52, 2001.

[22] Y. N. Zheng and Q. Liu, "Review of techniques for the mass flow rate measurement of pneumatically conveyed solids," Measurement, vol. 44, no. 4, pp. 589-604, 2011.

[23] O. Ochoa, A. A. Velasco, and C. Servin, "Towards a fast, practical alternative to joint inversion of multiple datasets: model fusion," Journal of Uncertain Systems, vol. 5, no. 2, pp. 119-136, 2011.

[24] M. P. Henry and D. W. Clarke, "The self-validating sensor: rationale, definitions and examples," Control Engineering Practice, vol. 1, no. 4, pp. 585-610, 1993. 


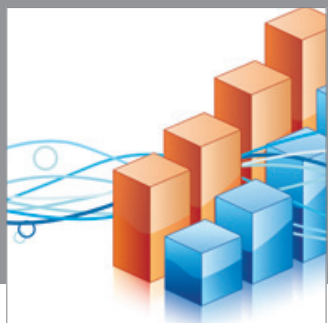

Advances in

Operations Research

mansans

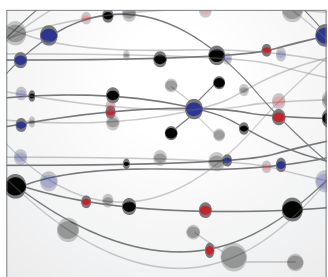

The Scientific World Journal
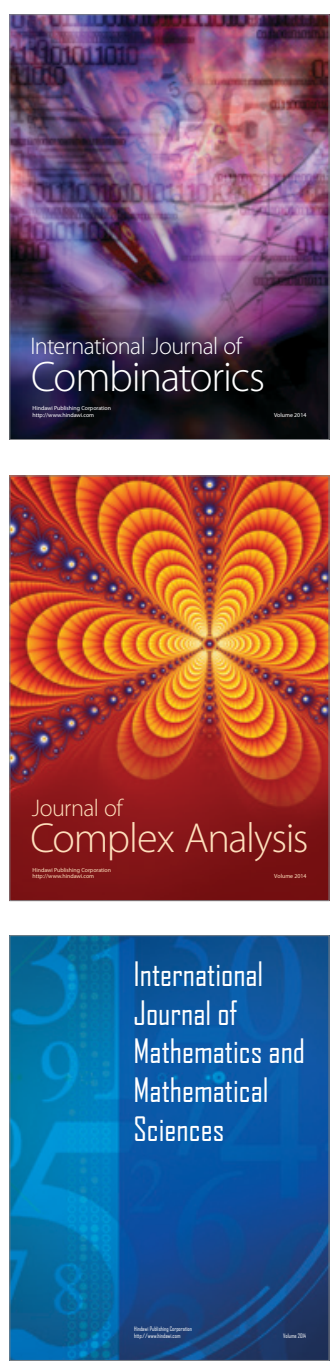
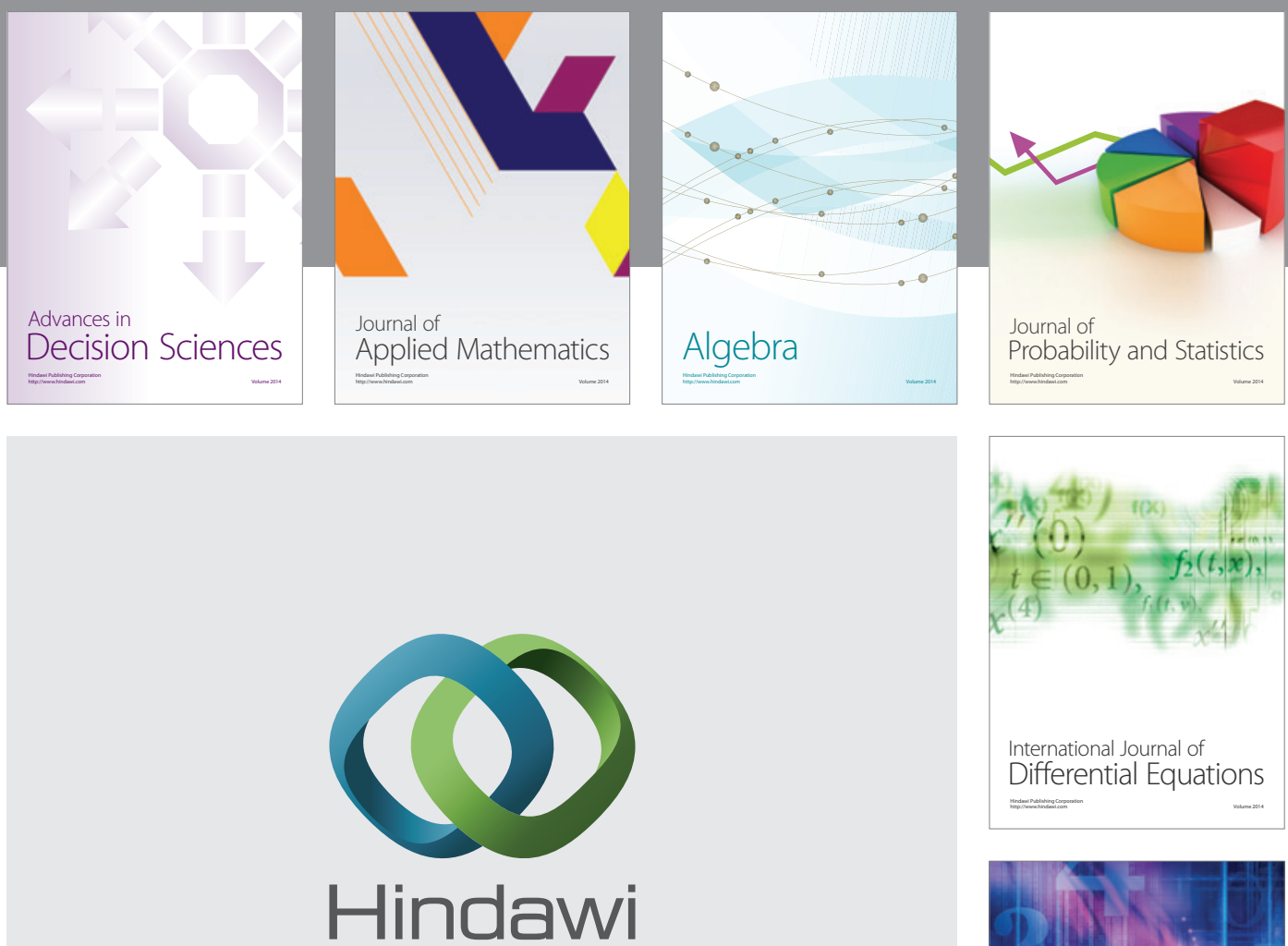

Submit your manuscripts at http://www.hindawi.com
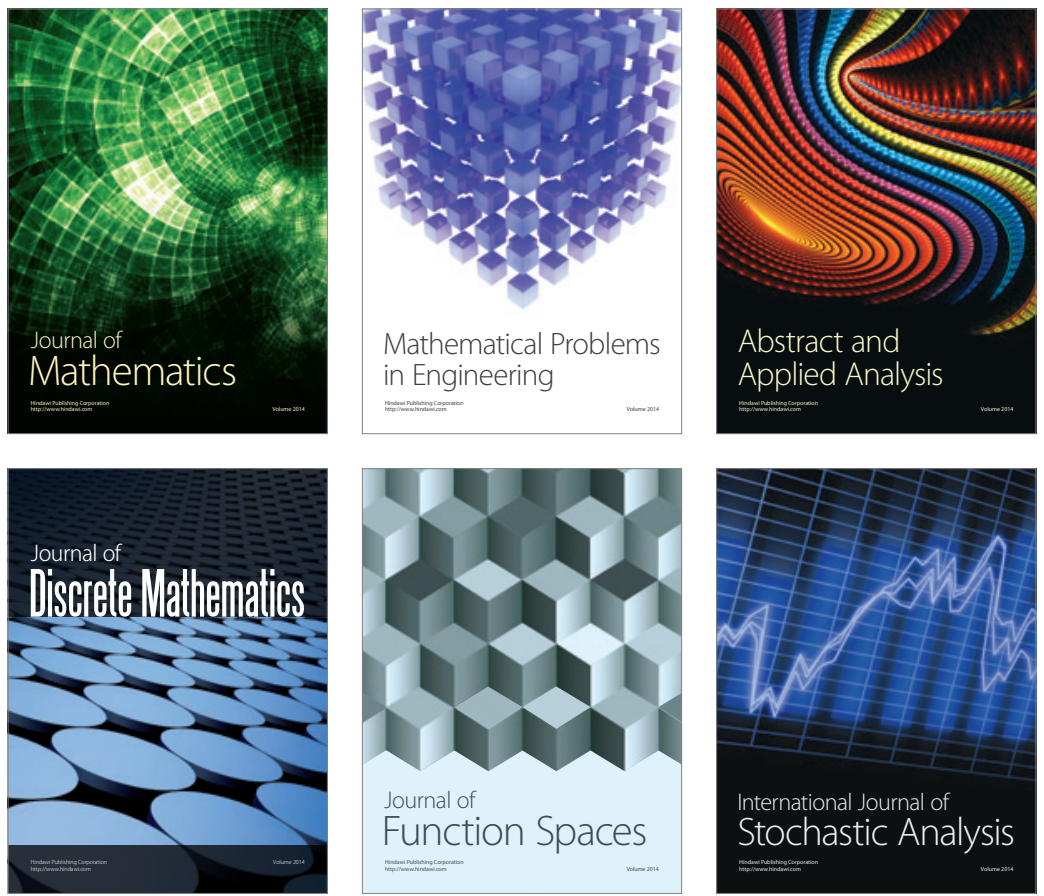

Journal of

Function Spaces

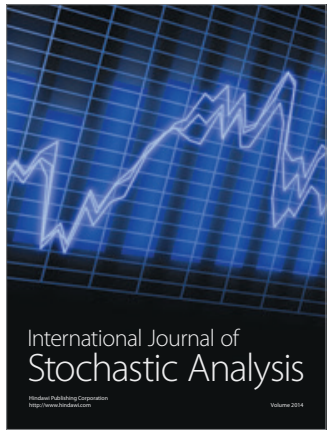

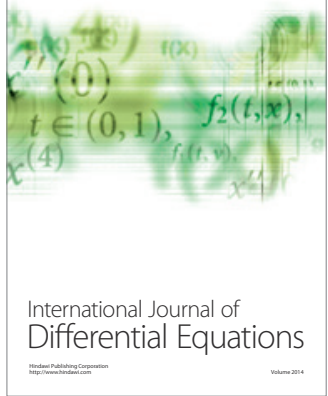
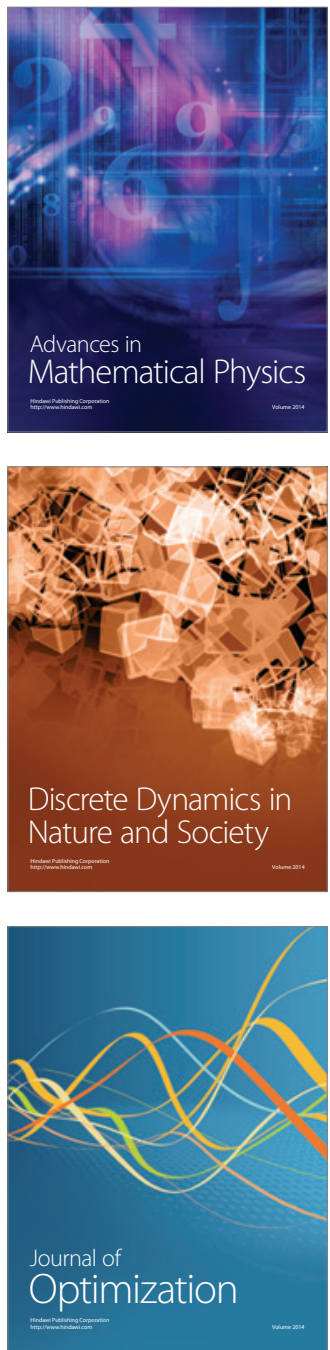